\title{
Self-Stabilizing Spoon for Parkinson’s Ailment
}

\author{
Jaswanth D K, Christopher Lenord J, Pavan S L \\ Student Dept. Of ECE, The National Institute of Engineering, Mysuru, India \\ E-mail: lenordjchristopher@gmail.com
}

\section{Nagaraju C}

Assistant professor, Dept. of ECE, The National Institute of Engineering, Mysuru, India

Received: 23 July 2020; Accepted: 06 September 2020; Published: 08 October 2020

\begin{abstract}
There is a substantial increase in the number of people getting affected by Parkinson Disease at the current scenario. Hence there is a critical need of support to such patients, as engineers it is our duty to ensure their needs as fulfilled. This paper basically proposes few models and prototypes that would bring a ray of hope in the patient's life. Here authors have proposed a supportive solution to this disorder. Basically our model uses a Nano board as the black box to which we provide the inputs and also we ensure the stabilizing in the form of tremor nullifying effect by using gyroscope, accelerometer and light weighted servo motors. In the present methodology self-stabilizing is done using servos using data provided to them by the gyro via a controller. Experimental analysis when carried out on different weight or object as an input on the spoon, designed model clearly depicts the decrease in the peak of tremor. The flexibility in the design basically refers to the all in one holder which is incorporated in the apex of the prototype, which aids the patient to not only use it is as a spoon, but also can be used to operate a key or any other objects by easily fitting it to the self-stabilizing device.
\end{abstract}

Index Terms: Actuators, Disorder, Parkinson, Patient, Stabilizing, Tremor nullifying.

\section{Introduction}

With the development of science and technology, our life relies on digital products much more than in previous decades, unfortunately the 2020 pandemic has reminded all of us about the importance of a healthy living.

In layman terms Parkinson disease is a Neurodegenerative disease which is generally found in aged persons which would actually limit the normal movement of a person by causing tremors during the movement of the gait.

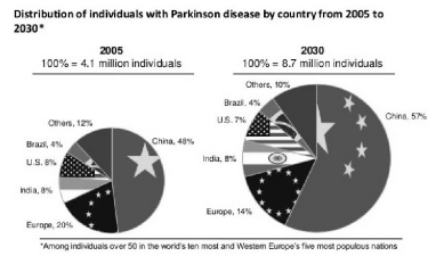

Fig.1. Distribution of Parkinson sufferers all over the globe. (Source: Neurology 2007; 68:384-6)

In the Fig.1. We can keenly observe that by the next decade i.e. 2030 the disorder would affect about approx. 9 million people across the globe. The fact which makes us to think about the disorder twice is that our country India would have $8 \%$ of the sufferers across the globe which is sums up to about 1 million approx. which is a number that cannot be overlooked. As of now there is lack of effective treatment for the disease which made us to think of developing a supportive equipment for the sufferers which would help them to lead a stable and independent life.

The medical devices group at different University have charged small research groups with developing devices related to certain diseases and impairments. The group of researchers, consisting of the authors of this document, has been tasked with developing a device related to Parkinson's disease (PD). No further description or static requirement has been given so it became necessary for the research group to identify a problem for which a device could aid in developing an aid or solution [4]. The precise development and operation of the PD monitoring device is described in the following sections. One such of equipment is a Self-stabilizing spoon which would primarily aid them in having their food without the support of others making them self-dependent.

In order to identify a means to create a device that would be of help to Parkinson patients, the paper comes up with a design that overcome some of the limitations that are present in the existing designs, the crucial limitation was that the peak tremor frequency varied from person to person because the people have natural tendency of tremor which can also 
depend on the gender, age, fitness, etc [6]. Due to this the design could not be generalized, so this paper gives the use of the IMU and filters that can be used to overcome the above mentioned limitation. The next section depicts the Literature survey which consists of more than two papers, which provided us a basic idea of designing a prototype. The following section is the proposed system where we talk about the different components chosen and also the reason for choosing them. The next section provides details of the development of this project. It includes the hardware and software development done, and also the experimental results obtained by the prototype developed. All these information are in explained in detailed in the upcoming section. The remaining section discusses the future scope and then concludes with a summary of the prototype developed.

\section{LITERATURE SURVEY}

[1]Design of Mems Gyroscope to Detect and Monitor Parkinson's disease - A Study authored by Adhavi Shri.A.S, S.Praveen Kumar T.Aravind had proposed the idea of design a MEMS based gyroscope. The gyroscope they had developed, was capable of maintaining and measuring the orientation and it worked based on the principle of angular momentum. Here they had depicted that MEMS (Micro Electro Mechanical System) has the platform for this kind of studies[7]. One of the drawbacks of the design is it is not cost-effective for batch processing and the Nano scale could be used in the near future, extending the field to Nano-Electro-Mechanical-Systems(NEMS). In this paper, MEMS gyroscope structure was explained and analyzed. This gave us an insight on the working and calibration of a MEMS gyroscope which is the input device of our project.

[2]Interpersonal Synchrony-based Dynamic Stabilization Of the Gait Rhythm between Human and Virtual Robot Clinical Application to Festinating Gait of Parkinson's Disease Patient - A study authored by H. Uchitomil, K. Suzukil, T. Nishil, M. J. Hove, Y. Wada, S. Orimo, Y. Miyakel. They had proposed the idea of dynamic stabilization of a gaits rhythm using virtual Robot Moreover, they had developed an interpersonal emulation synchronous robotic gait. This was a biped virtual robot synchronized along with the Patient's footstep rhythm while walking together. They had also implemented the same with a Parkinson sufferer which showed rhythm disturbances during walking or movement of the gait. Their results showed that the festinating gait, evaluated by stride time reduction rate, significantly stabilized and accelerated less with the prototype they had developed compared to unassisted walking. One of the main limitation of the developed system was that it depicted a design of a whole arm which was very difficult to be used by the patient, and moreover we were up to the design of a light weight and compact spoon. The portion of this project was the stride and tremor detection analysis which was performed using Walk-Mate System. The valuable findings of this paper which was incorporated in our work is the process to quantify the tremor observed in a Parkinson person which is observed in the Fig.10.This includes some of the references [8-15].

[3]'sHaKe_n_SCALE': A Measurement system of Motor characteristics of Parkinson's Disease- A Study done Suprio Bhattacharya, Rahul Krishna, Brent Clay, and Christopher Moore. They had developed a Standard (UPDRS) way of measurement of tremors of a Parkinson person. They had designed a prototype system using Arduino and Inertial Measurement Unit, signal processing software and Graphical User Interface (GUI) in MATLAB to measure the different characteristics of motor movements in the Parkinson person. The Characteristics related to tremor gait, movements, finger tapping and pronation-supination were measured in the project. So, as a reference we can take a look of the results they had obtained which helped us a lot during the course of our project.

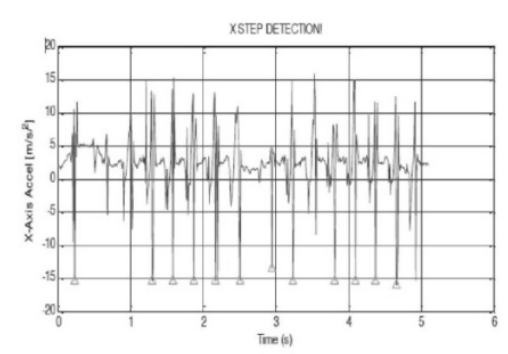

Fig.2. Step detection waveforms obtained in the paper 'sHaKe_n_SCALE'.

Finger-tapping measurements and gait measurements were done in this paper which helped us to choose the specific frequency range of operation required for a Parkinson person.

\section{PROPOSED SYSTEM}

In this paper we as engineers have tried to provide a support for a healthy living for the Parkinson Sufferers. Our proposed system is developed with sensor, controller and the actuator as a major part. 
A. SENSOR - It is a device that detects the changes in electrical or physical or other quantities and it produces an outcome which is an acknowledgement of changes taken place in the quantity. Some of the different sensors are proximity sensors, IR sensors, Temperature sensors etc. Out of those we have made use of inertial measurement sensor accompanied with accelerometer to measure the tremors of a Parkinson person.

B. CONTROLLER - A device that is a part of control system that generates control signals to reduce the deviation of the actual value from the desired value to almost zero or lowest possible value is a controller. For us in our module, in order to get accurate output the control action of the controller is responsible. The main controller which is used in this selfstabilizing spoon is the Arduino Nano. The reason for choosing the same is owing to its light weight, this property mattered a lot because we needed to make sure that the prototype is easily portable. In addition to that it has sufficient amount of memory and an optimum power consumption level.

C. ACTUATOR - A machine or part of a machine which moves or controls another part in response to an input that is provided is known as an actuator. These actuators are usually computer-controlled actuators and are mechanical devices that convert the output commands from the computer into mechanical action. The actuators that we have deployed in our project are servo-motors. The choosing of one of their kind was a trade of between weight and the maximum torque of the motor, this was again to optimize the portability of the device also ensuring no compromise in the required power to perform the desired movements.

The key features of Arduino Nano is that it is small, complete, and is user-friendly board. It is based on the ATmega328. It has a wide variety of ports such as Input/output options which includes Digital I/O, Analog I/O and also the PWM output pins. In the project we use the Arduino Nano as a black box, where it is used to control the different servo motors involved in the project by using the accelerometer and IMU (Inertial Measurement Unit) as the input devices. Given its low cost, low weight and versatility it was the ideal choice for the project, we could have gone for the other Arduino boards, but NANO board was a right choice because the main agenda of the project was the portability and ease of use, which directly depends on the compactness $(18$ x $45 \mathrm{~mm})$ and light weight $(7 \mathrm{~g})$.Moreover it provided a 8bit ADC which is more than enough to handle the raw data of the IMU which was in the same range of values.

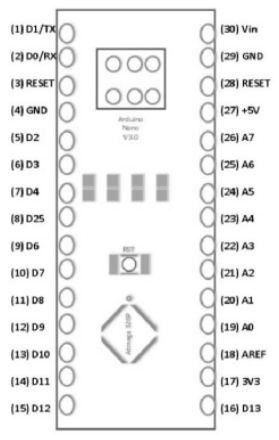

Fig.3. Pin diagram of Arduino Nano

The module GY-273 is a module which is based on the Honeywell HMC5883L IC. It is used for low-field magnetic sensing. It encompasses a digital interface for varieties of applications. Some of them are magnetometry, low cost compassing, etc. The HMC5883L is made up of series magneto-resistive sensors plus an ASIC containing amplification, offset cancellation, automatic degaussing strap drivers and a 12-bit ADC that enables 1 to 2 degree compass heading accuracy. The I2C serial bus is present for an easy interface. The fig. below depicts the pin outs of HMC5883L gyroscope.

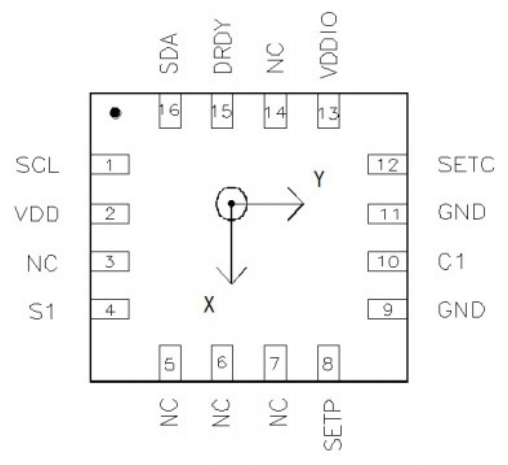

Fig.4. Pin diagram of HMC5883L

The key feature of analog HS-125MG is that it is a super thin servo. It measures "3.048 x 1.016 x 3.302 " $\mathrm{cm}$. Manoeuvrings the Hitec's MP Gear, which is the HS-125MG that is very durable and a perfect for gliders. The main 
reason of using a servo - motor in the place of widely available DC-motors is owing to the use case of our project whose working predominantly depends on the multidimensional angle constraints rather than just clockwise/anticlockwise directions as seen in the counterpart i.e. DC motors, in addition to this the torque and weight of the servo motor matched the project requirements which made it seldom negligible.

The basic block diagram shown in Fig.5. can deeply narrate the working of the system developed.

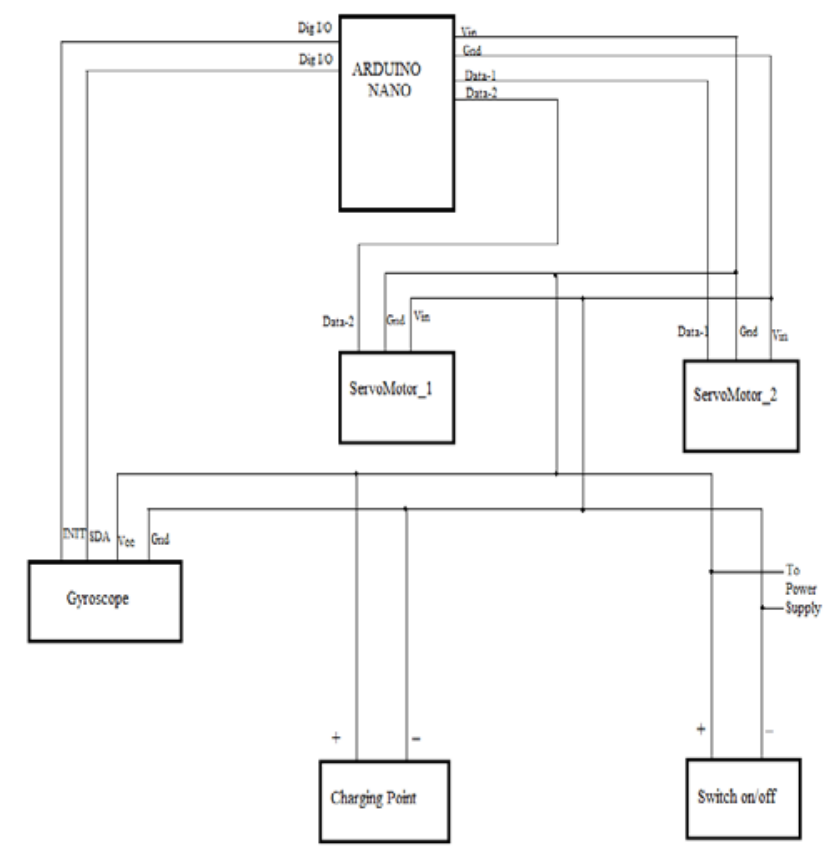

Fig.5. Circuit diagram of self-stabilizing spoon for Parkinson syndrome.

The main working goes in this way, basically the gyroscope acts an input device where we obtain the tremors from the patients hand i.e. we do obtain movements of the Parkinson person in two dimension i.e. X and Y axis. Now we obtain some analog values of the axial movements which is differentiated as axial movement and acceleration of the movement, since it's a 3 axis - GYRO we would have a total of 6 values of analog data.

Now this analog data is processed using the processing functions and is made available as a control signal in order to control the servo-motor. In principle the servomotors aroused in conjunction with one another.Servo- 1 is used for vertical movement and the latter for horizontal motion. Thus with the help of the control signal generated by the Nano board the motors are moved and the tremor cancellation is obtained. Now the tremor cancellation up to a desirable extent can be observed in the spoon. The process is summarized as shown in the Fig.6.

Rate of the gyroscope = ADC value from gyroscope-Zero value of the gyroscope)/sensitivity - (1). 


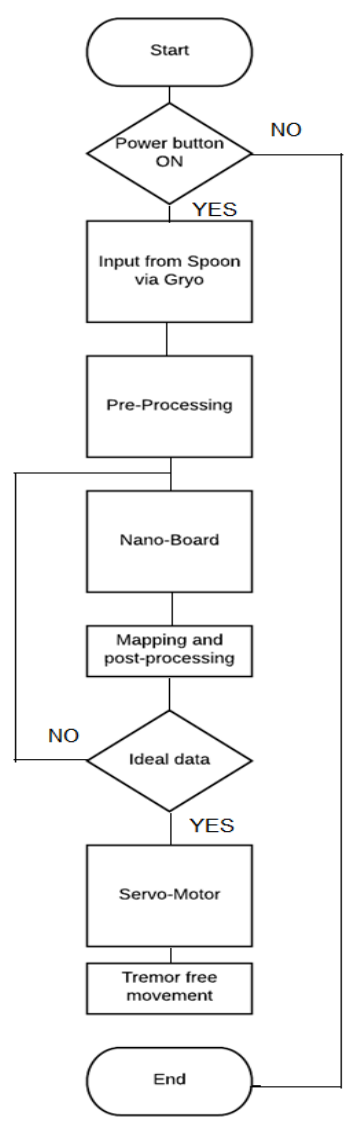

Fig.6. Flow chart of the Self-stabilizing Spoon for Parkinson person

\section{RESULTS AND DISCUSSIONS}

In the present method result are summarized through graphs and snapshots. A complete model developed by the authors is as shown in Fig. 7. When the handle is undergoing a pitch motion in the upward direction the spoon is tilted zero degrees below (vertical axis) and both the curves are coinciding. This phenomenon occurs because the spoon is following the direction opposite of the handle to easily pick up objects. In other words, instead of restricting hand tremors, the spoon (connected to motors) will follow the direction opposite to the handle (connected to sensor) and the user will be able to shovel up the desired object. One can experience that when the handle is tilting some degrees, the spoon bowl is stabilized around minus of same angle (at least close to that value). When undergoing roll movements, the spoon is turning from negative to positive when the handle is rolling from positive to negative. In order to simulate the hand tremors, the device was then tilted more rapidly. The corresponding results from the GYRO before and after pre-process are as shown in Fig.8. As a negative pitch motion is applied to the sensor i.e. a rear-downward motion say $25^{\circ}$, the spoon has a tendency to follow the handle to about $25^{\circ}$ or above until it stabilizes to $0^{\circ}$ or slightly above due to minute errors which is negligible as it may be less than $2^{\circ}$.

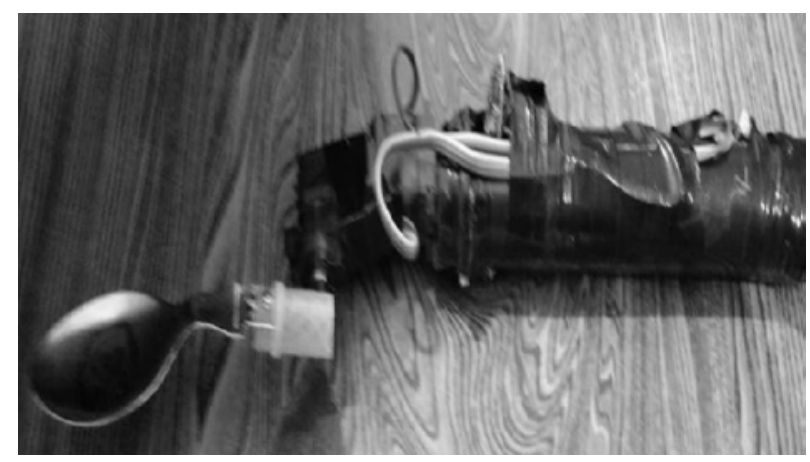

Fig.7. Hardware model of the Self-stabilizing Spoon for Parkinson person

In the Fig.8 and Fig.9 we can take a look at the plots which we had obtained at the GYRO end while performing the experiments. Firstly, the values of the magnetometer which we used was a 3-Dimensional one, basically we could measure 


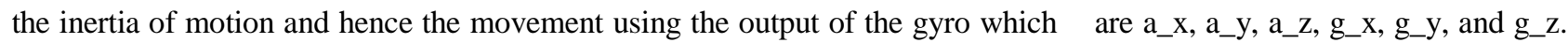
Here "a" refers to the accelerometer readings along the three dimensions and "g" indicated the same parameters in the view of gyroscope. The values obtained for the gyroscope in the different axes $x$ and y are in the range -65355 to +65355 (since 8 bit IMU) which is processed to -17000 to +17000 , which is considered as the ideal values in our case owing to the calibration and datasheet of the IMU. In the Fig.8 owing to the lack of processing we obtained a background noise and non-ideal values included readings, but upon the pre-processing we could get a more specific, spread out and close to ideal values which is depicted in the Fig.9.

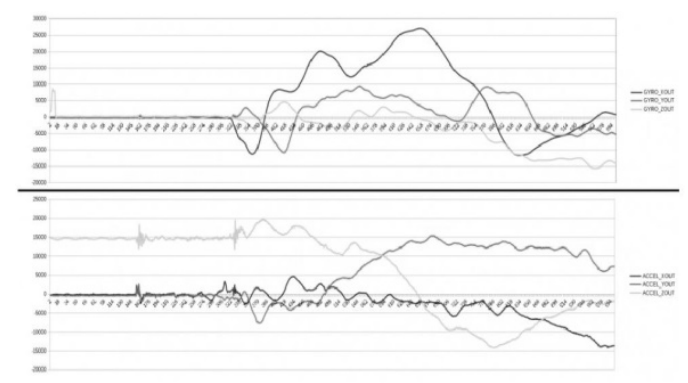

Fig.8. GYRO values before pre-processing v/s time.

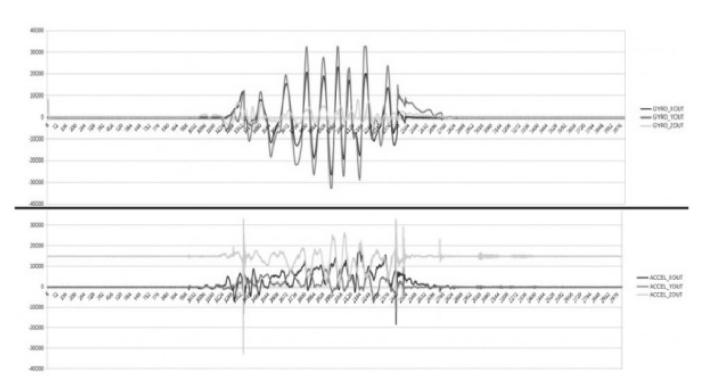

Fig.9. GYRO values after pre-processing v/s time.

Table 1. Table representing the gyro values and their angle mapping

\begin{tabular}{|c|c|c|c|c|}
\hline SL No. & Values from IMU & Angle in Radians & Angle in Degrees & Input Angle for Servo \\
\hline $\mathbf{1}$ & -17000 & 0 & 0 & 0 \\
\hline $\mathbf{2}$ & -12750 & 0.78539 & 45 & -45 \\
\hline $\mathbf{3}$ & -9916.67 & 1.3089 & 75 & -75 \\
\hline $\mathbf{4}$ & 0 & 1.57079 & 90 & -90 \\
\hline $\mathbf{5}$ & 9916.67 & 1.832 & 105 & -105 \\
\hline $\mathbf{6}$ & 12750 & 2.356 & 135 & -135 \\
\hline $\mathbf{7}$ & 17000 & 3.12 & 179 & -179 \\
\hline
\end{tabular}

The Table 1. is the experimental values the authors have obtained, this mapping follows the relation,

Angle (in radians) $\alpha$ [(data from IMU x $\pi$ ) /17000], when data from IMU $>0$ - (2)

Angle (in radians) $\alpha \pi-[($ data from IMU x $\pi$ ) / 17000], when data from IMU $<0$ - (3)

Here the values are obtained in radians and the same can be converted in degree which is used to control the servo motors, the value 17000 refers the zero error value or calibration value which is got through the calibration of the IMU and also the datasheet of the same. Thus we can observe the different angular values for the data obtained through the IMU, here the stable point for both the servos are 90 degree as mentioned in the table. This is the data which is mentioned in the previous section which is used to handle the servos. Basically here it is to observe that the IMU values obtained are derived from the library function oh header file MPU6050.h, which is dependent on the GYRO present in the IMU. After comparing the values obtained from the mapping function of the Servo (dependant on the servo library) and the values obtained using the relation in the eq- 2 and eq-3, we could conclude that the values are comparable with a accuracy of 0.01 units.

The data obtained using this setup can also be used for tremor analysis by neurologists, because one need to have all the possible information to provide a right treatment for the disease. 

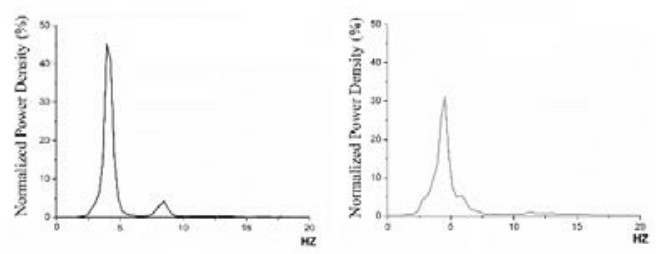

Fig.10. Plot of Normalized Power Density (\%v) vs frequency

Upon conducting the experiment on the Parkinson person and on a normal human being we obtained the readings through which we tried to plot a graph of normalized power density and the frequency of operation (Fig.10) which is nothing but the reciprocal of time we obtained the peak of $46.9 \mathrm{~mW} / \mathrm{m} 3$ at $4.4 \mathrm{~Hz}$ for a Parkinson person's movement before using a normal spoon (this was observed by using a gyro to a normal spoon) and when we obtained the readings using the self-stabilizing spoon which we had developed we could observe the decrease in tremor at the spoon end which estimated a peak of $30 \mathrm{~mW} / \mathrm{m} 3$ at about $4.7 \mathrm{~Hz}$. Basically this clearly depicts the decrease in the peak of tremor from 46.9 $\mathrm{mW} / \mathrm{m} 3$ to $30 \mathrm{~mW} / \mathrm{m} 3$ which is a significant decrease. Thus the normalized graphs give us the picture of the power distribution of the tremors observed during the hand movement of the Parkinson person and thus helped us to improve the filter design by deducing the frequency range, in this case it was from (0to 20Hz) as shown in the Fig.10.

\section{CONCLUSION AND FUTURE SCOPE}

Parkinson affected people suffer both physically due to the tremor observed in them and also mentally by being dependent on the others even to perform their primary life activities. So this work by the authors was implemented for Tremor cancellation of motor symptoms associated to a Parkinson's disease patient. The various parameters obtained such as the nullifying angle to be applied for the servo, frequency range of operation and the power distribution in the frequency range aided in the development of the prototype of the Self-stabilizing spoon for the Parkinson person. Thus it turns out to be a ray of hope of the Parkinson sufferers because the porotype is of light weight, compact, easy to use and flexible.

A room for improvement would be designing a more sensitive and auto-adjustable model that can be one time calibrated and used for different patients with a very minimum intervention of the medical specialists. Since care was taken in designing a generalized model which can be improved in the coming times to different equipments, to name a few selfstabilizing gloves for the Parkinson bloke where one could do all the actions using his hand with reduced tremors. This would turn out a ray of hope for a Parkinson bloke, also to design a walking sticks for the Parkinson patients, which can be extended from the same design.

\section{ACKNOWLEDGEMENT}

We wish to express our heartily gratitude to Dr. Rohini Nagapadma, Ph.D., Principal, The National Institute of Engineering, Mysuru, India, for encouraging us to complete this accomplishment. We would like to thank our parents and also the faculty of, The National Institute of Engineering, Mysuru, India, for their valuable ideas, blueprint and support throughout the completion.

We would like to express our sincere gratitude to KARNATAKA STATE COUNCIL FOR SCIENCE AND TECHNOLOGY, Indian Institute of Science campus (Bengaluru) for funding and helping us to pull off this work.

\section{References}

[1] Adhavi Shri.A.S, S.Praveen Kumar and T.Aravind “Design of Mems Gyroscope to Detect and Monitor Parkinson's disease” 2013 International Conference on Smart Structures \& Systems (JCSSS-20 13), March 28 - 29, 2013, Chennai, INDIA

[2]H. Uchitomil, K. Suzukil, T. Nishil, M. J. Hove, Y. Wada, S. Orimo, Y. Miyakel "Interpersonal Synchrony-based Dynamic Stabilization” Of the Gait Rhythm between Human and Virtual RobotClinical Application to Festinating Gait of Parkinson's Disease Patient Tokyo Institute of Technology, Midori, Yokohama 226-8502, Japan.

[3] Suprio Bhattacharya, Rahul Krishna, Brent Clay, and Christopher Moore “'sHaKe_n_SCALE’: A Measurement system of Motor characteristics of Parkinson's disease” IEEE VACS 2015

[4]Politis, M., Wu, K., Molloy, S., G Bain, P., Chaudhuri, K. R., \& Piccini, P. (2010). Parkinson's disease symptoms: the patient’s perspective.Movement Disorders: Official Journal of the Movement Disorder Society, 25, 16461651. doi:10.1002/mds.23135

[5] Duval, C. (2006). "The Rest and postural tremors in patients with Parkinson’s disease”. Brain Research Bulletin, $70,4448$. doi:10.1016/j.brainresbull.2005.11.010

[6] Wei Wang, Xiaoyong Lv, and Feng Sun, "Design of Micromachined Vibratory Gyroscope with Two Degree-of-Freedom DriveMode and Sense-Mode" Senior Member, IEEE, July 2012.

[7] Kavitha K. Nithya R, Shahana R K. Anju Gupta Alagappan M, "Design and Simulation of MEMS Based Gyroscope for Vestibular Prosthesis", COMSOL conference, 2012 
[8] A. Koenig, D. Novak, X. Omlin, M. Pulfer, E. Perreault, L. Zimmerli, M. Mihelj, and R. Riener. "Real-Time Closed-Loop Control of Load in Neurological Patients During Robot-Assisted Gait Training," IEEE Transactions on Neural Systems and Rehabilitation Engineering, vol. 19(4), 2011, pp.453-464.

[9] M. Montero-Odasso, H. Bergman, N. A. Phillips, C. H. Wong, N. Sourial, and H. Chertkow, "Dual-tasking and gait in people with Mild Cognitive Impairment. The effect of working memory," BMC Geriatrics, voI9(1), 2009, p.4l.

[10] 1. M. Hausdorff, N. B. Alexander, "Gait Disorders: Evaluation and Management," Informa Healthcare, 2005.

[11] M. H. Thaut, and M. Abiru, "Rhythmic Auditory Stimulation in rehabilitation of movement disorders: A review of the current research," Music Perception, vo1.27, 2010, pp.263-269.

[12] Politis, M., Wu, K., Molloy, S., G Bain, P., Chaudhuri, K. R., \& Piccini, P. (2010). Parkinsons disease symptoms: the patient's perspective. Movement Disorders : Official Journal of the Movement Disorder Society, 25, 16461651.

[13] J. Azulay, S. Mesure, B. Amblard, O. Blin, I. Sangla and J. Pouget, "Visual control of locomotion in Parkinson's disease," Brain, voI.l22(1), 1999, pp.III-120.

[14] 1. Azulay, S. Mesure, and o. Blin, "Influence of visual cues on gait in Parkinson's disease: Contribution to attention or sensory dependence?," Journal of the Neurological Sciences, voI.248(1-2), 2006, pp.I92-195.

[15] M. H. Thaut, C. G. Mcintosh, R. R. Rice, R. A. Miller, J. Rathbun, and 1. A. Brault, "Rhythmic auditory stimulation in gait training with Parkinson's disease patients," Movement Disorders, vol.ll, 1996, pp.193-200.

\section{Authors' Profiles}

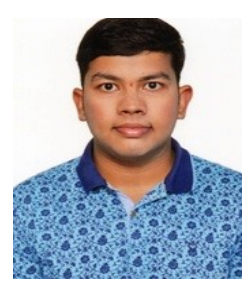

JASWANTH D K completed his B.E in Electronics and Communication from The National Institute of Engineering, Mysuru, currently he is working as Network Engineer, Cisco Systems Ltd.

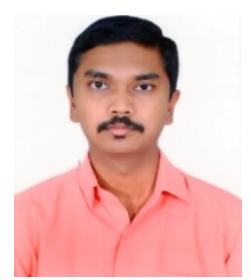

Christopher Lenord $\mathbf{J}$ completed his B.E in Electronics and Communication from The National Institute of Engineering, Mysuru, currently he is working as Associate Software Engineer at Robert Bosch Engineering and Business Solutions Ltd.

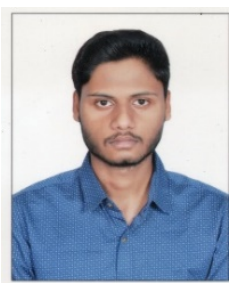

Pavan S L completed his B.E in Electronics and Communication from The National Institute of Engineering, Mysuru, currently he is working as Associate Engineer at L\&T Technology Services Ltd.

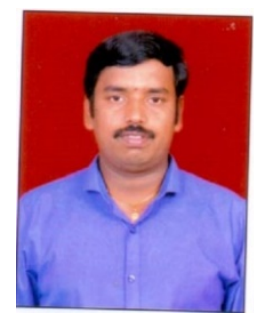

Dr. C Nagaraju completed his B.E and M-Tech from SJCE, Mysore. He has completed his Ph.D. at PET Research centre, Mandya. He has 15 years of teaching experience and currently working as Assistant Professor, in department of ECE at The National Institute of Engineering, Mysore. He has published more than 30 research publications in various National, International journals and Conference proceedings.

How to cite this paper: Jaswanth D K, Christopher Lenord J, Pavan S L, C Nagaraju, " Self-Stabilizing Spoon for Parkinson's Ailment ", International Journal of Engineering and Manufacturing (IJEM), Vol.10, No.5, pp38-44, 2020. DOI: 10.5815/ijem.2020.05.04 